\title{
Synthesis of a Zigzag Carbon Nanobelt
}

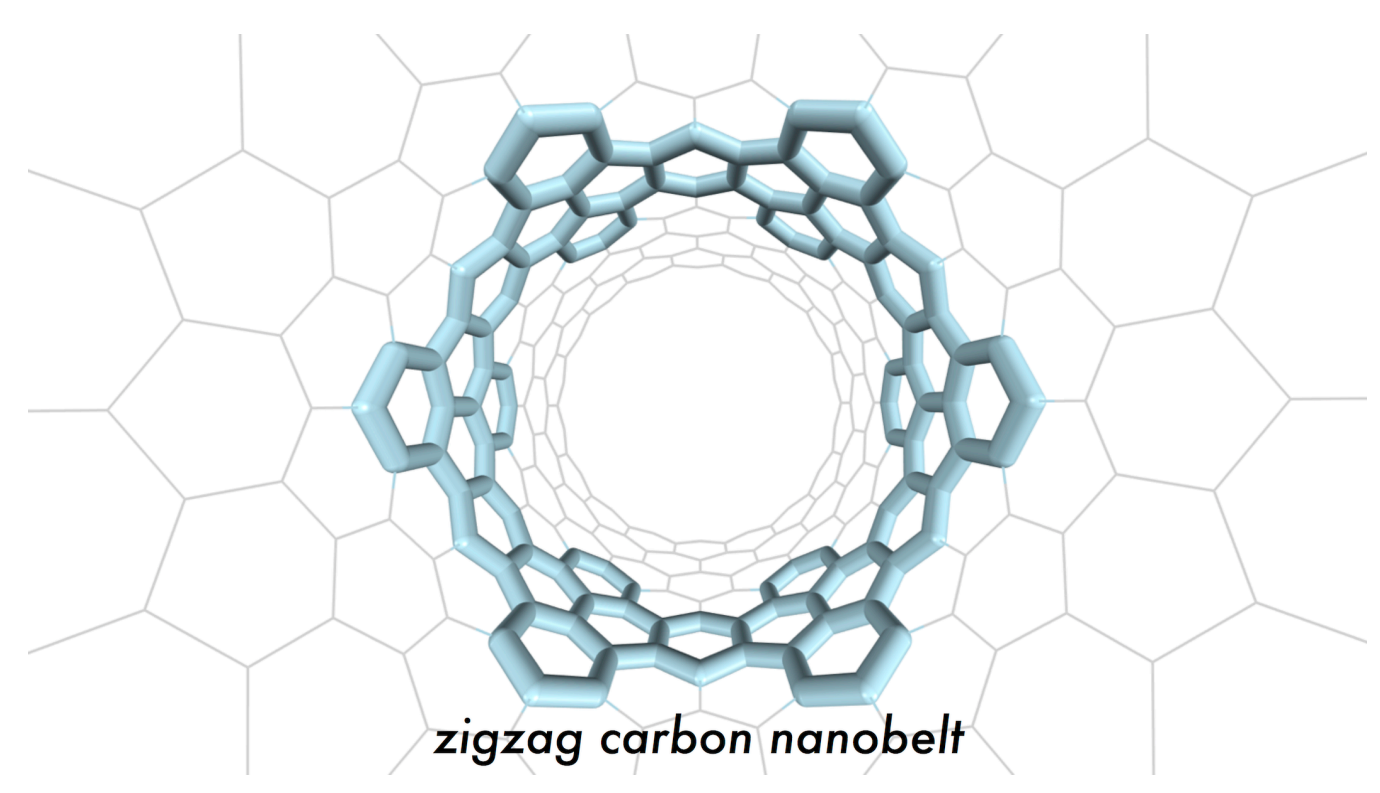

Kwan Yin Cheung, ${ }^{1}$ Kosuke Watanabe, ${ }^{2,3}$ Yasutomo Segawa, ${ }^{2,3,4,5, *}$ Kenichiro Itami ${ }^{1,2,4,6^{*}}$

1 Institute of Transformative Bio-Molecules (WPI-ITbM), Nagoya University, Chikusa, Nagoya 464-8602, Japan.

2 Graduate School of Science, Nagoya University, Chikusa, Nagoya 464-8602, Japan.

${ }^{3}$ Institute for Molecular Science, Myodaiji, Okazaki, 444-8787, Japan.

4 JST-ERATO, Itami Molecular Nanocarbon Project, Chikusa, Nagoya 464-8602, Japan.

${ }^{5}$ Department of Structural Molecular Science, SOKENDAI (The Graduate University for Advanced Studies), Myodaiji, Okazaki, 444-8787, Japan.

${ }^{6}$ Institute of Chemistry, Academia Sinica, Nankang, Taipei 115, Taiwan, R.O.C.

\section{Contact Information:}

Prof. Dr. Kenichiro Itami

Address: Institute of Transformative Bio-Molecules, Nagoya University, Chikusa, Nagoya 464-8602, Japan

Tel./Fax: +81-52-788-6098

E-mail: itami@chem.nagoya-u.ac.jp 


\section{Abstract:}

The structure-selective precise synthesis of carbon nanotubes (CNTs) has been long sought in materials science. The aromatic molecules corresponding to segment structures of CNTs, i.e. carbon nanobelts (CNBs), have been of interest as templates for CNT growth. Although two of three types, armchair and chiral CNBs, have been synthesized recently, zigzag CNBs remain elusive. Herein we report the synthesis and isolation of a zigzag CNB. The synthesis involves an iterative Diels-Alder reaction sequence followed by reductive aromatization of oxygen-bridged moieties. As predicted by theoretical calculations, this CNB was isolated as a stable compound. The structure of the zigzag CNB was fully characterized by X-ray crystallography, and its wide energy gap with blue fluorescence properties were revealed by photophysical measurement. With synthetic strategies towards all three types of CNBs in hand, the road to the precise synthesis of CNTs can now proceed to the next stage. 


\section{Main text}

The bottom-up chemical synthesis of belt-shaped fully fused polycyclic aromatic hydrocarbons has been of enduring interest to chemists for over 60 years. ${ }^{1-6}$ These structures have been named carbon nanobelts $(\mathrm{CNBs})^{7}$ as they represent a partial structure of carbon nanotubes (CNTs), discovered in $1991 .{ }^{8}$ CNBs have received a great deal of attention as a tool for the elucidation of the structural properties of CNTs at the molecular level, and also as a potential seed for the precise synthesis of CNTs. ${ }^{9,10}$ CNBs are classified by the CNT chiral index $(n, m)$ : armchair type $(n=m)$, chiral type $(n>m>0)$, and zigzag type $(m=0)$ (Fig. 1). Although the synthesis of CNBs involves tremendous difficulties due to their huge inherent strain, ${ }^{8}$ our group succeeded in the synthesis and isolation of an armchair CNB for the first time in 2017. ${ }^{11,12}$ In 2019, the group of Miao reported the synthesis of armchair and chiral CNBs. ${ }^{13}$ However, the synthesis and isolation of zigzag CNB, the last category, has not yet been achieved.

The simplest zigzag CNB is cyclacene (Figure 1b), the first proposed CNB by theoretical chemists. ${ }^{14}$ Cyclacene has no Clar aromatic sextet ${ }^{15}$ in its closed shell structure, and thus it is presumed to have a singlet biradical state by theoretical calculation. ${ }^{16}$ Not only because of its electronic state but also its strain energy, ${ }^{7}$ cyclacene is expected to have extremely high reactivity. In fact, many attempts towards the isolation of cyclacenes to date have failed, ${ }^{17-21}$ despite Wang and coworkers reported detection of a [8]cyclacene derivative by mass spectrometry. ${ }^{22}$ To resolve this issue, we previously proposed benzannulated cyclacenes as stable zigzag $\mathrm{CNBs}^{23}$ and synthetic attempts based on this molecular design were reported by Miao and coworkers. ${ }^{24,25}$ Although not included in the definition of CNBs, Tanaka and coworkers 
synthesized doubly stranded macrocyclic arenes bearing $-\mathrm{CH}_{2} \mathrm{O}-$ linkers, which correspond to armchair and zigzag CNTs. ${ }^{26,27}$

We now report on the first successful synthesis of a zigzag CNB by appropriate molecular design and synthetic strategy. The structure of the obtained zigzag CNB 1 was determined by X-ray crystallography to confirm that $\mathbf{1}$ has the sidewall segment of zigzag CNT with a chiral index of $(18,0)$. The measurement of optical properties of 1 revealed that 1 has a large energy gap coming from its closed shell state, which is in good agreement with the high stability of 1.

The zigzag CNB 1 was designed based on theoretical calculations. As we have previously reported, benzannulated cyclacenes are predicted to be stable owing to a closed shell structure and a wide energy gap. ${ }^{23}$ As shown in Figure 2a, the zigzag CNB 1 has a structure in which 12 benzene rings are fused to [18]cyclacene, a partial structure of $(18,0) \mathrm{CNT}$. Since all $\mathrm{sp}^{2}$ carbon atoms are contained in Clar sextets to form a fully benzenoid belt, high stability of $\mathbf{1}$ can be expected. Calculations were performed on 1 ', the unfunctionalized analogue of $\mathbf{1}$. From the anisotropy of the induced current density (ACID) plot $^{28}$ and nucleus-independent chemical shift $(\mathrm{NICS}(0))^{29}$ values shown in Fig. $2 \mathrm{~b}, \mathrm{c}$, rings $\mathrm{A}$ and $\mathrm{C}$ are aromatic Clar sextet whereas ring $B$ is nonaromatic. In addition, the strain energy of $1^{\prime}$ is $63.3 \mathrm{kcal} \cdot \mathrm{mol}^{-1}$ (see Supplementary Information (SI) for detail), which is slightly lower than that of [18]cyclacene $\left(73.6 \mathrm{kcal} \cdot \mathrm{mol}^{-1}\right) \cdot{ }^{7}$ According to the strain visualization obtained using StrainViz ${ }^{30}$ (Fig. 2d), the strain is applied almost equally to the circumferential bonds. From these electronic and structural analyses, it was presumed that $\mathbf{1}$ is a stable compound that could be isolated. 
The key synthetic strategy for 1 is the use of an oxanorbornadiene unit (a hexagonal ring bridged by an oxygen atom) as shown in Fig. 2 e, which can be regarded as an analog of the bending units used for the synthesis of cycloparaphenylenes (eq 1). ${ }^{31,32}$ Synthetic attempts of cyclacenes using oxanorbornadiene or oxanorbornene units were previously carried out, but product isolation could not be achieved, probably due to the instability of cyclacenes. ${ }^{17,18,21}$ Since similar belt-like compounds were detected by deoxygenation reaction from oxanorbornene units, ${ }^{33-35}$ this strategy was expected to be useful for the synthesis of stable zigzag CNBs such as 1.

The synthesis of 1 started with the reported 4,5,9,10-tetrabromo-2,7-di-tertbutylpyrene 3 (Fig. 3a). ${ }^{36}$ The reactive aryne generated from halogen-magnesium exchange reaction was trapped by furan and the subsequent retro-Diels-Alder reaction mediated by 3,6-di-2-pyridyl-1,2,4,5-tetrazine afforded 4 . Diels-Alder dimerization of $\mathbf{4}$ was induced in the presence of a small amount of $n$-BuLi ( 0.35 equiv of 4). The thus-obtained product was subjected to rhenium-catalyzed deoxygenative aromatization $^{37}$ to yield 5 in $54 \%$ based on unrecovered 4 , while $78 \%$ of 4 was recovered. After further transformation of $\mathbf{5}$ to $\mathbf{6}$ with furan, Diels-Alder reaction of $\mathbf{6}$ with the aryne derived from 3 and following retro-Diels-Alder reaction promoted by tetraphenylcyclopentadienone were performed to yield 7 in $75 \%$ yield. When the dimerization of $\mathbf{7}$ was initially attempted under the conditions used for the synthesis of $\mathbf{4}$ or $\mathbf{5}$, target macrocycle $\mathbf{2}$ was not detected in the resulting mixture. Fortunately, when hexafluorobenzene was added in expectation of a $\pi-\pi$ template effect ${ }^{38,39}$ (see Figs. S6-S8 in SI for details), the mass peak corresponding to 2 was observed by MALDITOF MS. Because of the complexity of the crude mixture, it was directly subjected to reduction conditions using low-valent titanium generated from $\mathrm{Cp}_{2} \mathrm{TiCl}_{2}$ and $\mathrm{Mn}$ powder, 
and after purification, the target zigzag CNB 1 was isolated in $5.4 \%$ yield over two steps. In the ${ }^{1} \mathrm{H}$ NMR spectrum of 1 , only three singlet signals at $9.01,8.73$, and 1.64 ppm were observed reflecting the highly symmetric structure of 1 . Zigzag CNB 1 is soluble in common halogenated and aromatic solvents such as dichloromethane, chloroform and toluene, and is stable as a solid and in solution under air and ambient light.

The structure of this zigzag belt was unambiguously characterized by X-ray crystallography. Suitable needle-like crystals were grown by slow diffusion of methanol into a solution of $\mathbf{1}$ in toluene. As shown in Fig. 3b, the belt-shaped structure with a zigzag-type sidewall can be clearly seen. The nanobelt shows a slight elliptic deformation with a diameter of about $14 \AA$ (Fig. 3c). Interestingly, 1 has tubular-like packing in the crystal structure as shown in Figs $3 c$ and $3 d$, with the voids occupied by 9 equivalents of disordered toluene molecules.

The diluted dichloromethane solution of 1 appears colorless with blue fluorescence upon irradiation with a UV light. The absorption and fluorescence spectra of $\mathbf{1}$ are shown in Fig 4a. The absorption features a band at around $336 \mathrm{~nm}$ and a small peak at $405 \mathrm{~nm}$, whereas the fluorescence spectrum shows two major peaks at $407 \mathrm{~nm}$ and $432 \mathrm{~nm}$ as well as a shoulder at $457 \mathrm{~nm}$. The absolute quantum yield and fluorescence lifetime of 1 were determined to be $10.3 \%$ and 9.25 ns, respectively, and the radiative $\left(k_{\mathrm{r}}\right)$ and nonradiative $\left(k_{\mathrm{nr}}\right)$ decay rate constants from the singlet excited state were determined accordingly $\left(k_{r}=1.6 \times 10^{6} \mathrm{~s}^{-1} ; k_{n r}=9.2 \times 10^{6} \mathrm{~s}^{-1}\right)$. Time-dependent density functional theory (TD-DFT) calculations of $1^{\prime}$ suggest that the $S_{0} \rightarrow S_{1}$ transition is symmetry forbidden and is attributed to HOMO-3 to LUMO transition. The $\mathrm{S}_{0} \rightarrow \mathrm{S}_{2}$ and $S_{0} \rightarrow S_{3}$ transition are degenerate and symmetry allowed (oscillator strength $(f)=$ 
0.0296 and 0.0295 ) and are related to HOMO-5 to LUMO and HOMO-4 to LUMO transitions. Thus, the weak absorption band at around $400 \mathrm{~nm}$ is assignable to $\mathrm{S}_{0} \rightarrow$ $\mathrm{S}_{1}$ forbidden transition and the small Stokes shift $\left(121 \mathrm{~cm}^{-1}\right)$ can be attributed to $0-0$ transition, which indicate a rigid $\pi$-conjugation structure of 1 similar to $(6,6) \mathrm{CNB} .{ }^{11}$

\section{Conclusions}

In summary, the first zigzag CNB 1 has been synthesized and fully characterized. Owing to the molecular design of a fully benzenoid CNB and a synthetic strategy using an oxanorbornadiene bending unit, 1 was isolated as a stable compound and its structure was characterized by X-ray crystallography. This synthesis represents a third viable method to synthesize CNBs in addition to the previously reported aryl-aryl coupling and Scholl reaction approaches. Given the recent renaissance of interest in CNBs and related belt-type nanocarbons, this work should inspire the future design and synthesis of CNBs and related frameworks with distinctive sidewall structures.

\section{References}

1 Kawase, T. \& Kurata, H. Ball-, bowl-, and belt-shaped conjugated systems and their complexing abilities: Exploration of the concave-convex $\pi-\pi$ interaction. Chem. Rev. 106, 5250-5273 (2006).

2 Tahara, K. \& Tobe, Y. Molecular loops and belts. Chem. Rev. 106, 5274-5290 (2006).

3 Eisenberg, D., Shenhar, R. \& Rabinovitz, M. Synthetic approaches to aromatic belts: Building up strain in macrocyclic polyarenes. Chem. Soc. Rev. 39, 28792890 (2010). 
4 Petrukhina, M. A. \& Scott, L. T. eds. Fragments of fullerenes and carbon nanotubes: designed synthesis, unusual reactions, and coordination chemistry (Wiley, 2012).

5 Segawa, Y., Yagi, A., Matsui, K. \& Itami, K. Design and synthesis of carbon nanotube segments. Angew. Chem. Int. Ed. 55, 5136-5158 (2016).

6 Segawa, Y., Ito, H. \& Itami, K. Structurally uniform and atomically precise carbon nanostructures. Nat. Rev. Mater. 1, 15002 (2016).

7 Segawa, Y., Yagi, A., Ito, H. \& Itami, K. A theoretical study on the strain energy of carbon nanobelts. Org. Lett. 18, 1430-1433 (2016).

8 lijima, S. Helical microtubules of graphitic carbon. Nature 354, 56-58 (1991).

9 Omachi, H., Nakayama, T., Takahashi, E., Segawa, Y. \& Itami, K. Initiation of carbon nanotube growth by well-defined carbon nanorings. Nat. Chem. 5, 572-576 (2013).

10 Sanchez-Valencia, J. R. et al. Controlled synthesis of single-chirality carbon nanotubes. Nature 512, 61-64 (2014).

11 Povie, G., Segawa, Y., Nishihara, T., Miyauchi, Y. \& Itami, K. Synthesis of a carbon nanobelt. Science 356, 172-175 (2017).

12 Povie, G., Segawa, Y., Nishihara, T., Miyauchi, Y. \& Itami, K. Synthesis and sizedependent properties of [12], [16], and [24]carbon nanobelts. J. Am. Chem. Soc. 140, 10054-10059 (2018).

13 Cheung, K. Y. et al. Synthesis of armchair and chiral carbon nanobelts. Chem 5, 838-847 (2019). 
14 Heilbronner, E. Molecular orbitals in homologen reihen mehrkerniger aromatischer kohlenwasserstoffe: I. Die eigenwerte yon Icao-mo's in homologen reihen. Helv. Chim. Acta 37, 921-935 (1954).

15 Clar, E. The aromatic sextet. Wiley (1972).

16 Chen, Z. et al. Open-shell singlet character of cyclacenes and short zigzag nanotubes. Org. Lett. 9, 5449-5452 (2007).

17 Kohnke, F. H., Slawin, A. M. Z., Stoddart, J. F. \& Williams, D. J. Molecular belts and collars in the making: A hexaepoxyoctacosahydro[12]cyclacene derivative. Angew. Chem. Int. Ed. Engl. 26, 892-894 (1987).

18 Ashton, P. R. et al. Molecular lego .1. Substrate-directed synthesis via stereoregular Diels-Alder oligomerizations. J. Am. Chem. Soc. 114, 6330-6353 (1992).

19 Cory, R. M., McPhail, C. L., Dikmans, A. J. \& Vittal, J. J. Macrocyclic cyclophane belts via double Diels-Alder cycloadditions: Macroannulation of bisdienes by bisdienophiles. Synthesis of a key precursor to an [8]cyclacene. Tetrahedron Lett. 37, 1983-1986 (1996).

20 Kintzel, O., Luger, P., Weber, M. \& Schlüter, A.-D. Ring-chain equilibrium between an [18]cyclacene derivative and a ladder oligomer. Eur. J. Org. Chem. 1998, 99105 (1998).

21 Schulz, F. et al. Exploring a route to cyclic acenes by on-surface synthesis. Angew. Chem. Int. Ed. 58, 9038-9042 (2019).

22 Shi, T.-H., Guo, Q.-H., Tong, S. \& Wang, M.-X. Toward the synthesis of a highly strained hydrocarbon belt. J. Am. Chem. Soc. 142, 4576-4580 (2020). 
23 Matsui, K., Fushimi, M., Segawa, Y. \& Itami, K. Synthesis, structure, and reactivity of a cylinder-shaped cyclo[12]orthophenylene[6]ethynylene: Toward the synthesis of zigzag carbon nanobelts. Org. Lett. 18, 5352-5355 (2016).

24 Wang, J. \& Miao, Q. A tetraazapentacene-pyrene belt: Toward synthesis of ndoped zigzag carbon nanobelts. Org. Lett. 21, 10120-10124 (2019).

25 Chen, H., Gui, S., Zhang, Y., Liu, Z. \& Miao, Q. Synthesis of a hydrogenated zigzag carbon nanobelt. CCS Chem. 2, 613-619 (2020).

26 Nishigaki, S. et al. Synthesis of belt- and Möbius-shaped cycloparaphenylenes by rhodium-catalyzed alkyne cyclotrimerization. J. Am. Chem. Soc. 141, 1495514960 (2019).

27 Nogami, J. et al. Enantioselective synthesis of planar chiral zigzag-type cyclophenylene belts by rhodium-catalyzed alkyne cyclotrimerization. J. Am. Chem. Soc. doi: 10.1021/jacs.0c03684 (2020).

28 Geuenich, D., Hess, K., Köhler, F. \& Herges, R. Anisotropy of the induced current density (ACID), a general method to quantify and visualize electronic delocalization. Chem. Rev. 105, 3758-3772 (2005).

29 Schleyer, P. v. R., Maerker, C., Dransfeld, A., Jiao, H. \& Hommes, N. J. R. v. E. Nucleus-independent chemical shifts: A simple and efficient aromaticity probe. J. Am. Chem. Soc. 118, 6317-6318 (1996).

30 Colwell, C. E., Price, T. W., Stauch, T. \& Jasti, R. Strain visualization for strained macrocycles. Chem. Sci. 11, 3923-3930 (2020).

31 Jasti, R., Bhattacharjee, J., Neaton, J. B. \& Bertozzi, C. R. Synthesis, characterization, and theory of [9]-, [12]-, and [18]cycloparaphenylene: Carbon nanohoop structures. J. Am. Chem. Soc. 130, 17646-17647 (2008). 
32 Takaba, H., Omachi, H., Yamamoto, Y., Bouffard, J. \& Itami, K. Selective synthesis of [12]cycloparaphenylene. Angew. Chem. Int. Ed. 48, 6112-6116 (2009).

33 Neudorff, W. D., Lentz, D., Anibarro, M. \& Schlüter, A. D. The carbon skeleton of the belt region of fullerene $\mathrm{C}_{84}\left(D_{2}\right)$. Chem. Eur. J. 9, 2745-2757 (2003).

34 Denekamp, C. et al. Towards a fully conjugated, double-stranded cycle: A mass spectrometric and theoretical study. Chem. Eur. J. 14, 1628-1637 (2008).

35 Standera, M. et al. Evidence for fully conjugated double-stranded cycles. Chem. Eur. J. 17, 12163-12174 (2011).

36 Franz, D., Robbins, S. J., Boeré, R. T. \& Dibble, P. W. Synthesis and characterization of 2,7-di(tert-butyl)pyreno[4,5-c:9,10- $\left.c^{\prime}\right]$ difuran and derived pyrenophanes. J. Org. Chem. 74, 7544-7547 (2009).

37 Murai, M., Ogita, T. \& Takai, K. Regioselective arene homologation through rhenium-catalyzed deoxygenative aromatization of 7-oxabicyclo[2.2.1]hepta-2,5dienes. Chem. Commun. 55, 2332-2335 (2019).

38 Bühner, M. et al. A novel type of cationic host molecules with r-acceptor properties. Angew. Chem. Int. Ed. Engl. 27, 1553-1556 (1988).

$39 \mathrm{Xu}, \mathrm{Y}$. et al. Highly strained, radially $\pi$-conjugated porphyrinylene nanohoops. J. Am. Chem. Soc. 141, 18500-18507 (2019).

\section{Acknowledgements}

This work was supported by the ERATO program from JST (JPMJER1302 to K.I.), the Funding Program for KAKENHI from MEXT (JP1905463 to K.I.; JP16K05771, JP19H02701, and JP19K22183 to Y.S.), and The Murata Science Foundation (Y.S.). K.Y.C. acknowledges the Croucher Foundation Fellowship program. K.W. 
acknowledges Special Inter-University Researcher program in Institute for Molecular Science. The authors thank Mr. R. Okude (Nagoya University) for assistance of X-ray crystallography, Dr. V. Gandikota, Dr. K. Matsui, and Mr. K. Okada (Nagoya University) for pioneering synthetic attempts, and Dr. lain A. Stepek for fruitful comments. Mass spectroscopy was supported by Toray Research Center, Inc. Calculations were performed using the resources of the Research Center for Computational Science, Okazaki, Japan. ITbM is supported by the World Premier International Research Center Initiative (WPI), Japan.

\section{Author Contributions}

K.I., Y.S., and K.Y.C. conceived the concept and directed the project. Y.S. performed the X-ray crystallography. K.Y.C. performed the experiments. K.Y.C. and K.W. performed the computational studies. All authors contributed in preparing the manuscript.

\section{Author Information}

Correspondence and requests for materials should be addressed to K.I. (itami@chem.nagoya-u.ac.jp) and Y.S. (ysegawa@nagoya-u.jp). 


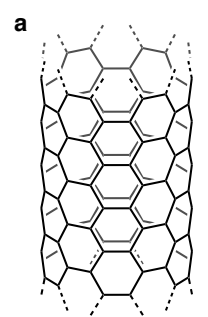

$(n, m)$ armchair CNT $n=m$

b

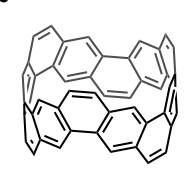

$(6,6)$ armchair CNB synthesized in 2017

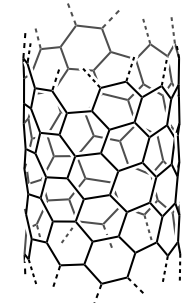

$(n, m)$ chiral CNT $n>m>0$

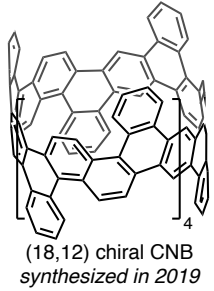

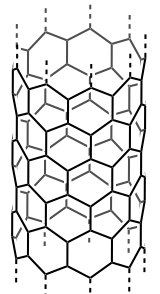

$(n, m)$ zigzag CNT $m=0$

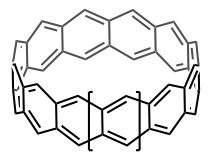

cyclacene proposed in 1954

Fig. 1. Carbon nanotubes and carbon nanobelts. (a) Structures of CNTs. (b) Structures of CNBs.

a

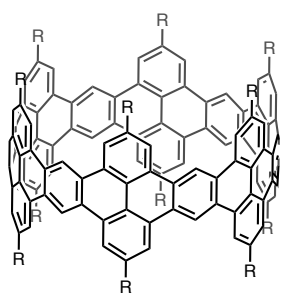

$(18,0)$ zigzag CNB
$1\left(\mathrm{R}={ }^{\mathrm{B}} \mathrm{Bu}\right), \mathbf{1}^{\prime}(\mathrm{R}=\mathrm{H})$

c

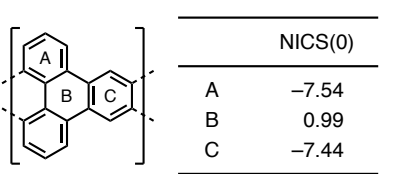

e
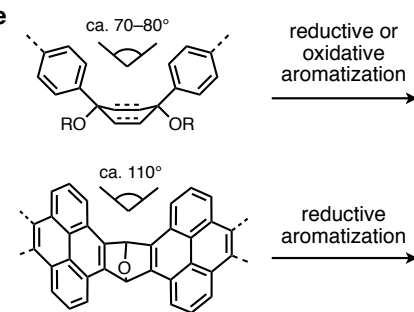

b

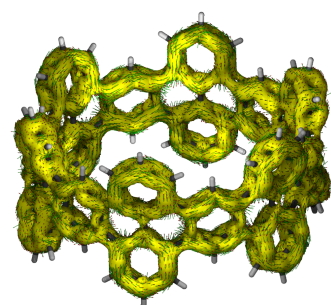

d
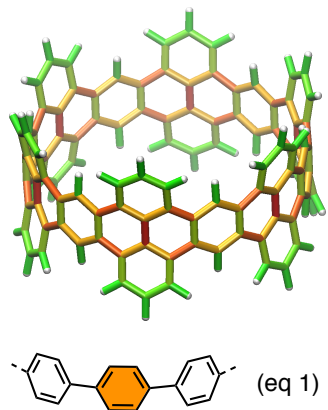

Fig. 2. Molecular design and synthetic strategy of zigzag carbon nanobelt. (a) Structures of 1 and 1'. (b) ACID plot of 1' (isovalue: 0.04). (c) NICS(0) values of the hexagonal rings of 1' calculated by using GIAO B3LYP/6-311+G(2d,p)//B3LYP/6-31G(d) level of theory. (d) StrainViz drawing of 1', where the bonds bearing high, middle, and low strain are shown as red, yellow, and green, respectively. (e) Synthetic strategies of cycloparaphenylenes (eq. 1) and 1 (eq. 2). 
(i,i)

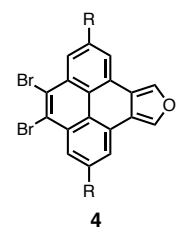

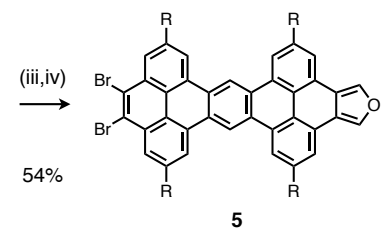
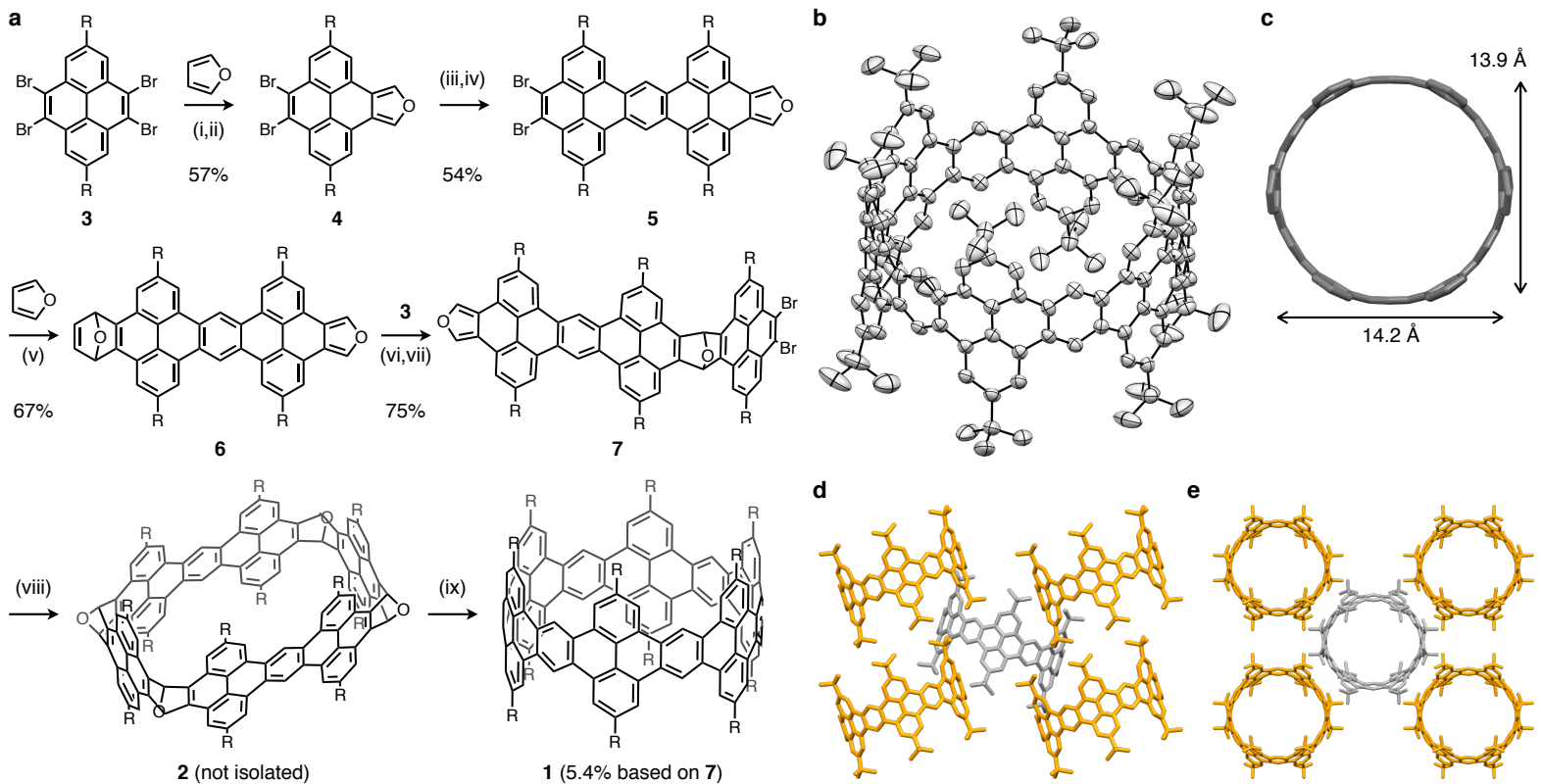

Fig. 3. Synthesis and structure of 1. (a) Synthesis of zigzag carbon nanobelt 1. Reagents and conditions: (i) furan (10 equiv.), $\mathrm{PrMgCl} \cdot \mathrm{LiCl}$ (1.1 equiv.), THF, $-46{ }^{\circ} \mathrm{C}$, overnight; (ii) 3,6-di-2-pyridyl1,2,4,5-tetrazine (1.05 equiv.), $\mathrm{CHCl}_{3}, 60^{\circ} \mathrm{C}$, overnight; (iii) $n$-BuLi $\left(0.35\right.$ equiv.), toluene, $-78{ }^{\circ} \mathrm{C}, 2 \mathrm{~h}$; (iv) $\mathrm{P}(\mathrm{OPh})_{3}$ (1.5 equiv.), $\mathrm{NH}_{4} \mathrm{ReO}_{4}(5 \mathrm{~mol} \%), \mathrm{Na}_{2} \mathrm{SO}_{4}$ (1 equiv.), toluene, $100{ }^{\circ} \mathrm{C}$, overnight; (v) furan (50 equiv.), $n$-BuLi (1.3 equiv.), $\mathrm{PhCl} / \mathrm{THF},-78{ }^{\circ} \mathrm{C}, 1.5 \mathrm{~h}$; (vi) 3 (4 equiv.), $i-\operatorname{PrMgBr}$ (3.5 equiv.), THF, $0{ }^{\circ} \mathrm{C}, 3 \mathrm{~h}$; (vii) tetraphenylcyclopentadienone (1.05 equiv.), toluene, $130{ }^{\circ} \mathrm{C}$, overnight; (viii) hexafluorobenzene (5 equiv.), $n$-BuLi (4 equiv.), PhCl/toluene, $-46{ }^{\circ} \mathrm{C}, 1 \mathrm{~h}$; (ix) $\mathrm{Cp}_{2} \mathrm{TiCl}_{2}$ (10 equiv.), Mn powder (50 equiv.), THF, $60{ }^{\circ} \mathrm{C}$, overnight. (b) ORTEP drawing of 1 with $50 \%$ thermal probabilities; hydrogen atoms and solvent molecules are omitted for clarity. (c) Diameters of 1. (d,e) Packing structure of 1.
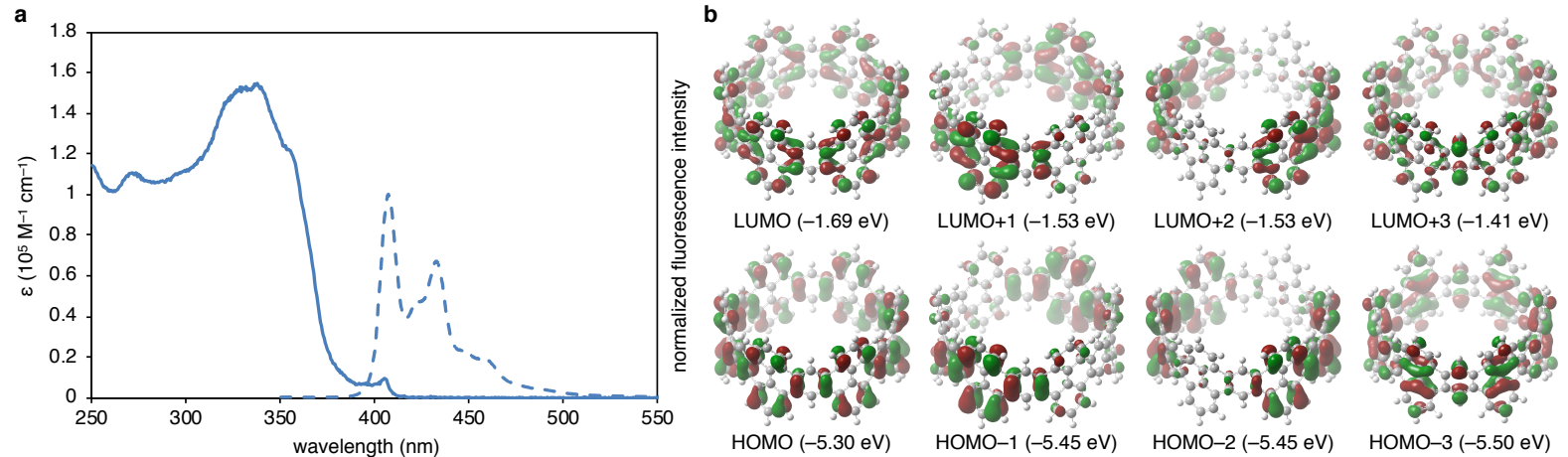

Fig. 4. Photophysical properties of 1. (a) UV-vis absorption (solid line) and fluorescence (broken line) of the dichloromethane solution of 1. (b) Frontier molecular orbitals of 1' and their energies, calculated by using B3LYP/6-31G(d) level of theory (isovalue: 0.04). 


\section{[Manuscript Information]}

Title: Synthesis of a Zigzag Carbon Nanobelt

Authors: Kwan Yin Cheung, Kosuke Watanabe, Yasutomo Segawa, and Kenichiro Itami

The number of words in the paper: 146 (abstract) +1370 (main text) +271 (legends in total) +924 (references)

The number of references: 39

The number of figures/tables: 4 figures, comprising 14 separate panels in total

Postal address: Prof. Kenichiro Itami, Institute of Transformative Bio-Molecules, Nagoya University, Chikusa, Nagoya 464-8602, Japan

Phone/Fax: +81-52-788-6098

E-mail: itami@chem.nagoya-u.ac.jp 